\title{
Modelos matemáticos para crescimento e produção de peso de creme comestível de palmito, em árvores individuais de Euterpe edulis Mart.
}

\author{
JEFERSON L. G. WENDLING ${ }^{1}$ \\ Carlos Roberto S Anquetta $^{2}$ \\ SÉrgio Ahrens ${ }^{3}$
}

\begin{abstract}
RESUMO
Este trabalho consiste em recomendar modelos matemáticos para representar o crescimento e a produção, de variáveis dimensionais, especialmente o peso de creme de palmito em função da idade, para árvores de Eurtepe edulis Mart.. Os dados foram obtidos em 110 árvores, com idades conhecidas, plantadas a céu aberto em propriedades rurais e urbanas, nos municípios de Antonina, Guaraqueçaba e Morretes, no Estado do Paraná. Para estimar a produção do Peso de Creme, primeiro as variáveis de fácil mensuração foram feitas função da idade, obtendo-se em seguida as predições para o Peso de Creme, a partir do DAP e da Altura de Estipe (estimados), ajustando-se os dados à equação $\mathrm{Y}=$ b.DAP + c. $\left(\mathrm{DAP}^{2} . \mathrm{H}_{\text {estipe }}\right)$. Para a série de dados estudada, conclui-se que: o Modelo de Gompertz foi o mais apropriado para representar o crescimento e a produção em função da Idade, e a utilização deste modelo em plantios comerciais de palmito, poderá representar satisfatoriamente o crescimento propiciando estimativas realistas para a produção desejada de creme de palmito.

Palavras-chave: creme de palmito, crescimento, Euterpe edulis, modelo matemático, produção

\footnotetext{
${ }^{1}$ Eng $^{\circ}$ Florestal da Diretoria de Fiscalização Ambiental do Instituto Ambiental do Paraná, exBolsista da CAPES e Mestrando do Curso de Pós-Graduação em Engenharia Florestal da UFPR

${ }^{2}$ Eng $^{\text {o }}$ Florestal, M. Sc., Dr., Professor do Departamento de Ciências Florestais e do Curso de Engenharia Florestal da UFPR, Bolsista do CNPq

${ }^{3}$ Eng $^{\text {o }}$ Florestal, M. Sc., Dr., Pesquisador em Biometria e Manejo Florestal do Centro Nacional de Pesquisas em Florestas da EMBRAPA.
} 


\section{ABSTRACT \\ Growth and yield models for the edible heart of the Euterpe edulis}

Mart. palm tree. This paper shows mathematical models to express the growth and yield of the edible part of the Brazilian heart palm tree Euterpe edulis Mart.. Data to fit the models came from 110 sample trees with age known and planted under full light conditions in rural and urban areas of Antonina, Guaraqueçaba and Morretes counties, eastern Paraná State, southern Brazil. To estimate yield in terms of the edible part of the palm trees, firstly the easily measurable variables were made functions of tree age and converted to give predictions directly from $\mathrm{DBH}$ and stem height by using the following equation: $\mathrm{Y}=$ b.DBH + c. $\left(\mathrm{DBH}^{2} \cdot \mathrm{H}_{\text {stem }}\right)$. The most suitable equation was selected by analyzing the best of fit for the data set used in this study. The model of Gompertz was that best represented the growth and yield of the heart palm tree as a function of tree age. The selected model can be considered satisfactory and useful in predicting the edible part of the heart palm tree and, therefore, it can be recommended for its estimation.

Key words: heart of palm, growth, Euterpe edulis, mathematical model, yield.

\section{INTRODUÇÃO}

O interesse econômico pela espécie Euterpe edulis Mart. concentra-se na parte comestível da palmeira, que produz fina iguaria denominada palmito, também chamada de creme. Segundo ROSSETI et al. (1986), no período entre 1980 a 1984, a produção de palmito em conservas foi de 100.000 t anuais, e no mercado internacional verificou-se um preço crescente, correspondendo a US\$ 1.990/t em 1985, apresentando tendência a alcançar até US\$2.200/t em 1986. FERREIRA \& PASCOALINO (1986) observaram que no período de 1973 a 1983, a exportação do palmito em conservas passou de 4.416 para 10.971 toneladas. O valor da exportação, em dólar FOB, foi de US\$ 3.539 .000 a US\$ 27.020.000, em 1983.

As contribuições apresentadas por ROSSETI et al. (1986) e FERREIRA \& PASCOALINO (1986) indicaram uma tendência crescente para a produção de conserva de palmito, entretanto, a exploração de E. edulis tradicionalmente ocorre em populações naturais, sendo realizada sob a forma de extrativismo, muitas vezes predatório e também clandestino.

Embora importantes contribuições tenham sido apresentadas, principalmente as documentadas por KIRCHNER et al. (1987) e FANTINI et. al. (1997), no que tocante ao ajuste de modelos matemáticos para a predição da produção de palmito, na atualidade praticamente inexistem instrumentos de análise que permitam a obtenção de estimativas de produção de creme de palmito, quer quando plantado sob a cobertura da vegetação natural, quer quando cultivado em condições de plena insolação, e que possam viabilizar esforços de análise econômico-financeira da silvicultura de E. edulis, com o propósito de determinar a sua melhor utilização. 
Este trabalho tem por finalidade representar o crescimento e a produção, de variáveis dimensionais, e especialmente para o peso de creme comestível de árvores individuais do palmiteiro nativo da Mata Atlântica, Euterpe edulis Mart..

\section{MATERIAL E MÉTODOS}

Os dados utilizados para a realização da pesquisa, são provenientes de 110 palmeiras plantadas a céu aberto (isoladas ou em pequenos povoamentos), entre os anos de 1976 a 1995, portanto com as idades conhecidas, situadas em propriedades rurais e urbanas localizadas nos municípios de Antonina, Guaraqueçaba e Morretes, no Estado do Paraná.

A coleta de dados foi realizada durante o mês de julho/97. Em cada palmeira selecionada, mediram-se as variáveis consideradas como de fácil obtenção (CAP, altura total e da estipe), enquanto a idade foi determinada através de informação prestada diretamente pelo proprietário. Em seguida, procedeu-se o corte das palmeiras para mensurar as variáveis de difícil obtenção ou destrutivas (peso da cabeça, comprimento da cabeça, diâmetro inferior e superior da cabeça, diâmetro do creme, comprimento do creme, peso bruto do creme, peso do creme comestível). Não foram cortadas 19 árvores (com idade de 2 e 3 anos), por não apresentarem produção de creme de palmito. A Figura 1 ilustra todas as partes da palmeira e as respectivas variáveis dimensionais empregadas nesta pesquisa.

O peso bruto e o peso de creme comestível de palmito, resultantes de cada palmeira, foram medidos em balança com precisão de 1 grama. $\mathrm{Na}$ separação entre a porção comestível e a parte fibrosa, adotou-se o padrão de qualidade estabelecido pela Indústria e Comércio de Conservas Florestas, com sede no município de Antonina, onde estas atividades foram executadas. As atividades e os produtos desta indústria são representativas das demais fábricas na região.

Para melhor compreensão da Figura 1, a seguir são descritas as variáveis empregadas neste estudo. Idade é o número expresso em anos, do espaço de tempo decorrido desde o plantio de cada palmeira até 1997; DAP é o diâmetro da estipe, em centímetros, medido na altura de $1,30 \mathrm{~m}$ a partir do nível do solo; Altura Total é a altura em metros, medida desde o nível do solo até a inserção da primeira folha; Altura da Estipe é a altura do caule da palmeira, em metros, medida desde o nível do solo até o início do colmo; Comprimento da Cabeça é o comprimento em centímetros, da bainha interna do colmo, que envolve o creme de palmito, ou seja, a porção comestível e não comestível (fibrosa); Diâmetro da Cabeça é o diâmetro, em centímetros, medido nas extremidades da cabeça de palmito. Definiu-se como Diâmetro Inferior da Cabeça, o diâmetro localizado na parte inferior do colmo (próximo à estipe) e por Diâmetro Superior da Cabeça, o diâmetro localizado na parte superior de colmo (próximo às folhas); Peso da Cabeça é o peso total, em gramas, da 
cabeça de palmito; Diâmetro do Creme é o diâmetro, em centímetros, da porção comestível do creme de palmito, medido na extremidade inferior. Comprimento do Creme é o comprimento do creme, em centímetros, correspondente à sua porção comestível; Peso Bruto do Creme é o peso total do creme, em gramas, incluindo-se as partes comestível e fibrosa, obtida logo após o descascamento da cabeça; Peso do Creme Comestível é o peso, em gramas, da porção comestível do palmito, obtida após a separação da porção fibrosa. Os diâmetros das variáveis mencionadas, foram obtidos dividindo-se suas respectivas circunferências por $\pi$.

Para possibilitar a estimativa do peso de creme comestível de palmito, desenvolveram-se duas etapas consecutivas de análise quantitativa, conforme descrito na sequiência:

- Na primeira, foram obtidas as estimativas para as variáveis de fácil mensuração (DAP, altura total e altura da estipe) em função da idade, ajustando-se os dados mensurados em cada variável, ao modelo de crescimento com a seguinte estrutura funcional:

\section{DAP; altura total; altura de estipe $=f(\mathrm{I})$}

Nesta etapa foram testados os seguintes modelos (Tabela 1):

Tabela 1 - Modelos matemáticos testados para ajuste dos dados de crescimento e produção de palmito

Table 1 - Growth models tested for the hearth palm tree

\begin{tabular}{ll}
\hline \multicolumn{1}{c}{ Modelo } & \multicolumn{1}{c}{ Função Matemática } \\
\hline Chapman-Richards & $\mathrm{Y}=\mathrm{a} \cdot(1-\exp (-\mathrm{b} . \mathrm{I}))^{\circ}$ \\
Prodan & $\mathrm{Y}=\frac{\mathrm{I}^{2}}{\mathrm{a}+\mathrm{b} \cdot \mathrm{I}+\mathrm{c} \cdot \mathrm{I}^{2}}$ \\
Gompertz & $\mathrm{Y}=\mathrm{a} \cdot \exp (-\mathrm{b} \cdot \exp (-\mathrm{c} . \mathrm{I}))$ \\
\hline
\end{tabular}

onde: $\quad \mathrm{Y}=$ variável dependente;

$\mathrm{I}=$ idade (anos);

$\mathrm{a}, \mathrm{b}, \mathrm{c}=$ coeficientes da equação.

Os coeficientes e os indicadores de ajuste correspondentes $\left(\mathrm{F}, \mathrm{R}^{2}{ }_{\text {ajustado }}\right)$ foram calculados através do pacote estatístico Statgraphics Plus - Versão 7.1, e o do Erro Padrão da Estimativa (Syx \%), calculado pelo programa Excel (Microsoft).

Selecionou-se, como mais apropriado, o modelo matemático que obteve os melhores resultados para os indicadores de ajuste (maior valor do teste de F, maior $\mathrm{R}^{2}$ e menor Syx \%), melhor distribuição gráfica dos resíduos e os valores estimados mais coerentes com os observados, ou seja, em relação às médias calculadas em cada idade. 
- Na segunda etapa, as estimativas do peso de creme comestível de palmito foram obtidas a partir das variáveis de fácil mensuração (DAP, altura total e altura da estipe) estimadas em função da idade, cujos valores observados para o peso comestível, foram ajustadas ao modelo matemático com a seguinte estrutura funcional:

PC CREME $=f($ DAP, altura da estipe $)$

A equação utilizada para o ajuste aos dados de peso de creme comestível, foi a equação de biomassa $\mathrm{Y}=\mathrm{b} \cdot \mathrm{DAP}+\mathrm{c} \cdot\left(\mathrm{DAP}^{2} \cdot \mathrm{H}_{\mathrm{Estipe}}\right)$, na forma reduzida (sem o intercepto), selecionada como a mais apropriada para a predição de biomassa, para o conjunto de dados considerado. Os coeficientes e os respectivos indicadores de ajuste $\left(\mathrm{F}, \mathrm{R}^{2}\right.$ ajustado) foram calculados pelo pacote estatístico Statistica versão 5, e o do Erro Padrão da Estimativa (Syx\%), calculado pelo programa Excel (Microsoft).

A opção pela altura de estipe em detrimento da altura total, como variável independente do modelo matemático, deve-se à pequena diferença entre a correlação destas variáveis com o peso do creme comestível. Pondera-se ainda, que a altura da estipe pode ser mensurada com menor susceptibilidade de erros.

Figura 1 - Perfil esquemático do palmiteiro e suas partes componentes Figure 1-Schematic profile of a heart palm tree and its components

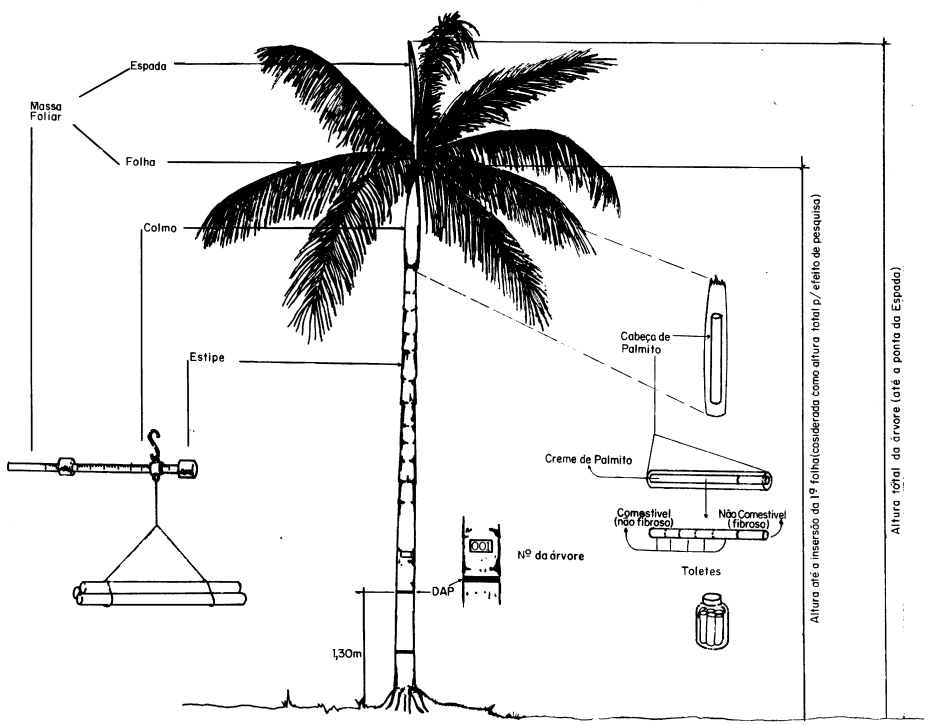


Também efetuou-se o ajuste dos modelos crescimentos (ChapmanRichards, Gompertz e Prodan) aos dados, para a obtenção das estimativas de Peso de Creme Comestível diretamente em função da idade, e os resultados foram comparados aos do modelo de biomassa.

Considerando o interesse na obtenção de estimativas para Diâmetro do Creme de palmito, adotaram-se os seguintes procedimentos:

- O ajuste do modelo de crescimento, para a predição do Diâmetro de Creme em função da idade,

- A predição do diâmetro do creme em função do DAP (que é a variável mais facilmente mensurável em uma árvore), ajustando-se o modelo linear simples $\mathrm{Y}$ $=\mathrm{a}+\mathrm{b} . \mathrm{X}$ (equação da reta) aos dados. A opção pela equação da reta, fundamenta-se no fato de que para o conjunto de dados, verificou-se uma relação com tendência linear entre o DAP e o diâmetro do creme. Os coeficientes e indicadores de ajuste foram calculados pelo pacote estatístico Statistica versão 5, e o Erro Padrão da Estimativa (Syx \%), calculado pelo programa Excel (Microsoft).

\section{RESULTADOS E DISCUSSÃO}

Os coeficientes calculados para os Modelos de Chapman-Richards, Prodan e de Gompertz, e seus respectivos indicadores de ajuste são apresentados na Tabela 2.

De acordo com os resultados obtidos, os indicadores de ajuste calculados para o Modelo de Chapman-Richards e Gompertz apresentaram resultados levemente melhores em termos de indicadores de ajuste em relação ao Modelo de Prodan. Porém, entre ambos não houve praticamente nenhuma diferença. $\mathrm{O}$ mesmo ocorreu em relação à distribuição dos resíduos absolutos. 
Tabela 2 - Modelo de Gompertz: coeficientes e indicadores de ajuste para valores observados em DAP, altura total e em altura de estipe em função da idade

Table 2 - The Gompertz model: coefficients and goodness of fit statistics for tree DBH, total height and stem height as functions of tree age

\begin{tabular}{|c|c|c|c|c|c|c|}
\hline \multicolumn{7}{|c|}{ Modelo de Gompertz / Gompertz model } \\
\hline \multirow{2}{*}{$\begin{array}{c}\text { Variável } \\
\text { Dependente / } \\
\text { Dependent } \\
\text { variable }\end{array}$} & \multicolumn{3}{|c|}{ Coeficientes / Coefficients } & \multicolumn{3}{|c|}{$\begin{array}{c}\text { Indicadores de Ajuste / Goodness } \\
\text { of fit }\end{array}$} \\
\hline & $\mathbf{a}$ & $\mathbf{b}$ & $\mathbf{c}$ & $\mathbf{F}$ & $\begin{array}{c}\mathbf{R}_{\text {ajustado }}^{2} / \\
\text { Adjusted } \mathbf{R}^{2}\end{array}$ & Syx\% \\
\hline DAP & 12,075419 & 138,062516 & 1,397219 & 941,979 & 0,790199 & 21,91 \\
\hline A. Total & 7,8765447 & 11,4589384 & 0,6042230 & 451,665 & 0,654468 & 31,80 \\
\hline A. Estipe & 6,7507340 & 10,5336979 & 0,5315201 & 344,973 & 0,648971 & 37,40 \\
\hline \multicolumn{7}{|c|}{ Modelo de Prodan / Prodan model } \\
\hline DAP & 2,73901239 & $-0,44883148$ & 0,09738819 & 742,244 & 0,736446 & 24,56 \\
\hline A. Total & 5,54324942 & $-0,64022060$ & 0,14120509 & 457,214 & 0,648345 & 31,62 \\
\hline A. Estipe & 9,01640935 & $-1,10879331$ & 0,17907847 & 339,120 & 0,643513 & 37,69 \\
\hline \multicolumn{7}{|c|}{ Modelo de Chapman-Richards / Chapman-Richards model } \\
\hline DAP & 12,07687100 & 1,38938100 & 132,359629 & 942,028 & 0,790210 & 21,91 \\
\hline A. Total & 8,12498914 & 0,41306433 & 4,62630442 & 1589,634 & 0,659516 & 31,57 \\
\hline A. Estipe & 6,81151911 & 0,47795159 & 7,75288828 & 1099,359 & 0,650319 & 37,33 \\
\hline
\end{tabular}

Os indicadores de ajuste e a distribuição dos resíduos resultantes do Modelo de Prodan embora tenham sido similares aos obtidos pelos modelos de Gompertz e Chapman-Richards, este modelo apresentou tendência decrescente para o valores assintóticos da curva, isto é, a partir de determinada idade as estimativas diminuíram (decresceram ao longo do tempo), ao invés de aumentarem ou então permaneceram estáveis, o que não é admissível para as variáveis em estudo.

Os resultados dos indicadores de ajuste para os modelos analisados seguiram padrão semelhante no tocante às variáveis DAP, altura total e altura da estipe estimadas em função da idade, observando-se, também nestes casos, distribuição de resíduos homogênea.

Ambos os modelos, o de Gompertz e o de Chapman-Richards, foram satisfatórios para descrever o crescimento de árvores individuais de Euterpe edulis plantadas a céu aberto. Contudo, por motivos de facilidade de ajuste preferiu-se recomendar prioritariamente o modelo de Gompertz neste trabalho, o que não implica que o outro modelo também não possa ser empregado com bons resultados em plantios semelhantes aos deste estudo. Assim, para efeito de ilustração, compararam-se as estimativas obtidas aos seus respectivos valores médios observados em cada idade (Tabela 3), onde constatou-se que as predições eram coerentes com as observações. O mesmo pode-se constatar a partir dos Anexos deste artigo, os quais mostram os resíduos absolutos das variáveis analisadas a partir do modelo de Gompertz. Este aspecto sugere, portanto, que o modelo de Gompertz pode ser indicado como apropriado para modelar o crescimento e a produção de árvores individuais de Euterpe edulis Mart., tanto em DAP como em alturas total e da estipe. 
Tabela 3 - Valores médios observados e valores estimados pelo modelo Gompertz

Table 3 - Mean observed and predicted values by the Gompertz model

\begin{tabular}{|c|c|c|c|c|c|c|}
\hline \multicolumn{7}{|c|}{ Variável } \\
\hline \multirow{2}{*}{$\begin{array}{c}\text { Idade } \\
\text { (anos) / } \\
\text { Age } \\
\text { (years) }\end{array}$} & \multicolumn{2}{|c|}{$\mathrm{DAP}(\mathrm{cm}) / \mathrm{DBH}$} & \multicolumn{2}{|c|}{$\begin{array}{c}\text { Altura Total (m) / Total } \\
\text { height }\end{array}$} & \multicolumn{2}{|c|}{$\begin{array}{c}\text { Altura de Estipe (m) / } \\
\text { Stem height }\end{array}$} \\
\hline & $\begin{array}{c}\text { Valor Médio } \\
\text { Observado / } \\
\text { Observed mean } \\
\text { value }\end{array}$ & $\begin{array}{c}\text { Estimado } \\
\text { Gompertz/ } \\
\text { Predicted Gompertz }\end{array}$ & $\begin{array}{c}\text { Valor Médio } \\
\text { Observado/ } \\
\text { Observed mean } \\
\text { value }\end{array}$ & \begin{tabular}{|c|} 
Estimado \\
Gompertz/ \\
Predicted Gompertz
\end{tabular} & $\begin{array}{c}\text { Valor Médio } \\
\text { Observado/ } \\
\text { Observed mean } \\
\text { value }\end{array}$ & $\begin{array}{c}\text { Estimado } \\
\text { Gompertz/ } \\
\text { Predicted Gompertz }\end{array}$ \\
\hline 2 & 0,4 & 0,0 & 0,99 & 0,26 & 0,11 & 0,18 \\
\hline 3 & 1,3 & 1,5 & 1,03 & 1,21 & 0,29 & 0,80 \\
\hline 4 & 7,7 & 7,2 & 2,71 & 2,83 & 1,85 & 1,92 \\
\hline 5 & 10,2 & 10,6 & 4,96 & 4,51 & 3,86 & 3,23 \\
\hline 6 & 12,7 & 11,7 & 5,12 & 5,80 & 3,93 & 4,37 \\
\hline 7 & 10,4 & 12,0 & 7,06 & 6,67 & 595 & 5,23 \\
\hline 8 & 10,9 & 12,1 & 6,38 & 7,19 & 5,23 & 5,81 \\
\hline 9 & 11,4 & 12,1 & 7,06 & 7,49 & 5,62 & 6,18 \\
\hline 11 & 11,5 & 12,1 & 6,62 & 7,76 & 5,46 & 6,55 \\
\hline 12 & 11,6 & 12,1 & 7,00 & 7,81 & 5,85 & 6,63 \\
\hline 13 & 12,6 & 12,1 & 8,30 & 7,84 & 7,10 & 6,68 \\
\hline 15 & 13,0 & 12,1 & 9,80 & 7,87 & 8,60 & 6,73 \\
\hline 16 & 13,5 & 12,1 & 9,55 & 7,87 & 8,20 & 6,74 \\
\hline 17 & 10,6 & 12,1 & 7,31 & 7,87 & 6,27 & 6,74 \\
\hline 18 & 15,4 & 12,1 & 8,00 & 7,88 & 6,56 & 6,75 \\
\hline 19 & 12,8 & 12,1 & 7,79 & 7,88 & 6,60 & 6,75 \\
\hline 20 & 12,7 & 12,1 & 7,55 & 7,88 & 6,33 & 6,75 \\
\hline 21 & 13,2 & 12,1 & 8,65 & 7,88 & 7,25 & 6,75 \\
\hline
\end{tabular}

Pela análise dos valores contidos na Tabela 3 , verificou-se que o crescimento em diâmetro foi acelerado até a idade de 5 anos, diminuindo sua velocidade até a idade de 7 anos, quando então, atingiu a estabilidade. Para a altura total e a altura da estipe, o crescimento foi acelerado até a idade de 11 anos, sendo que a partir desta idade o crescimento foi lento, tornando as curvas suaves até atingirem a estabilidade.

As comparações entre os resultados obtidos, para os indicadores de ajuste referentes aos modelos ajustados para a predição de peso de creme comestível em função do DAP e da altura de estipe (Modelo de Biomassa), e também para predição de Peso de Creme comestível em função da Idade (Modelo de Gompertz), tem por finalidade permitir a avaliação do ajuste de cada modelo matemático aos dados, e não em selecionar o melhor, haja vista que tratam-se de procedimentos diferentes para a predição da variável dependente. Os coeficientes e os respectivos indicadores de ajuste, calculados para os modelos, são apresentados na Tabela 4.

Nos dois modelos, o valor de $\mathrm{F}$ foi significativo e o resultados do Syx \% foram idênticos, entretanto, o valor do $\mathrm{R}^{2}$ ajustado é indicativo de que a regressão através do modelo de biomassa, está propiciando melhor ajuste em relação ao modelo de Gompertz. A distribuição dos resíduos foi homogênea, em ambos os casos (ver Anexo). 
Tabela 4 - Coeficientes e indicadores de ajuste para estimativas peso de creme comestível de palmito: modelo de biomassa e modelo de Gompertz

Table 4 -Coefficients and goodness of fit of the biomass model and the Gompertz model for the edible heart palm weight

\begin{tabular}{|c|c|c|c|c|c|c|}
\hline \multicolumn{7}{|c|}{ Modelo de Biomassa / Biomass model } \\
\hline \multirow{2}{*}{$\begin{array}{c}\text { Variável Dependente / } \\
\text { Dependent variable }\end{array}$} & \multicolumn{3}{|c|}{ Coeficientes/Coefficients } & \multicolumn{3}{|c|}{$\begin{array}{l}\text { Indicadores de Ajuste/ } \\
\text { Goodness of fit }\end{array}$} \\
\hline & $\mathbf{a}$ & $\mathbf{b}$ & $\mathbf{c}$ & $\mathbf{F}$ & $\begin{array}{l}\mathbf{R}_{\text {ajustado }}^{2} / \\
\text { Adjusted } \mathbf{R}^{2}\end{array}$ & Syx\% \\
\hline $\begin{array}{l}\text { Peso de creme } \\
\text { comestível / Heart palm } \\
\text { weight }\end{array}$ & - & 16,18824166 & 0,353126 & 313,462 & 0,85032 & 47,84 \\
\hline \multicolumn{7}{|c|}{ Modelo de Gompertz / Gompertz model } \\
\hline \multirow{2}{*}{$\begin{array}{c}\text { Variável } \\
\text { Dependent variable }\end{array}$} & \multicolumn{3}{|c|}{ Coeficientes } & \multicolumn{3}{|c|}{ Indicadores de Ajuste } \\
\hline & $\mathbf{a}$ & b & $\mathbf{c}$ & $\mathbf{F}$ & $\begin{array}{l}\mathbf{R}_{\text {ajustado } /}^{2} \\
\text { Adjusted } R^{2}\end{array}$ & Syx\% \\
\hline $\begin{array}{l}\text { Peso de creme } \\
\text { comestível / Heart palm } \\
\text { weight }\end{array}$ & 557,4402 & 10,500390 & 0,532655 & 204,000 & 0,54531 & 47,36 \\
\hline
\end{tabular}

Através das curvas de produção obtidas pelos modelos de Biomassa e de Gompertz (Figura 2), verifica-se que o aumento do peso de creme comestível de palmito foi acelerado até a idade de 11 anos, e a partir desta idade a produção tornou-se estável. Ressalta-se que a curva de produção oriunda do modelo foi obtida utilizando-se como variáveis independentes, o DAP e a altura de estipe estimadas em função da idade, através do modelo de Gompertz.

Comparando-se os valores médios observados para o peso de creme comestível com os valores preditos pelos modelos de Biomassa e de Gompertz, na Tabela 4, observa-se que as estimativas estão mais coerentes para as idades mais jovens, enquanto que nas idades mais avançadas, principalmente a partir de 13 anos, verifica-se maior variação dos valores observados em relação às estimativas. Tal fato pode ser atribuído à necessidade de uma amostra maior ou a influências do sítio. Ainda, as estimativas obtidas pelo modelo de Gompertz, foram ligeiramente superiores em relação às observações e às preditas através do modelo de Biomassa, principalmente nas idades mais jovens.

No Estado do Paraná, a legislação disciplina o corte do palmito estabelecendo o diâmetro mínimo de $2,5 \mathrm{~cm}$ para o creme. Por esta razão, o modelo de Gompertz foi também, ajustado aos dados observados para o diâmetro do creme, objetivando estimar idade mínima de corte da palmeira.

Para estimar o diâmetro do creme em função da idade, obteve-se os seguintes coeficientes para o modelo de Gompertz: $\mathrm{a}=4,217679 ; \mathrm{b}=$ 468,046799 e $\mathrm{c}=-1,678512$. Os resultados dos indicadores de ajuste foram: $\mathrm{F}=$ 1008,$34 ; \mathrm{R}^{2}=0,8334$ e Syx $\%=21,97$ e os resíduos para as estimativas do diâmetro do creme, distribuíram-se de forma homogênea (ver Anexo), indicando que houve bom ajuste do modelo de Gompertz aos dados, e, portanto este foi apropriado para a predição do diâmetro do creme de palmito em Euterpe edulis Mart.. 
Tabela 5 - Valores médios observados e valores estimados pelos modelos de biomassa e de Gompertz

Table 5 - Mean observed and predicted values of edible heart palm weight predicted by the biomass and the Gompertz models

\begin{tabular}{c|c|c|c}
\hline \multirow{2}{*}{$\begin{array}{c}\text { Idade (anos) / } \\
\text { Age (years) }\end{array}$} & \multicolumn{3}{|c}{ Peso de Creme Comestível de Palmito (g) } \\
\cline { 2 - 4 } & Média Observada / & \multicolumn{2}{|c}{ Modelo / Model } \\
\cline { 3 - 4 } Observed mean & 0 & 0 & Biomassa / Biomass \\
\hline 2 & 0 & 25 & 15 \\
\hline 3 & 134 & 152 & 160 \\
\hline 4 & 335 & 300 & 268 \\
\hline 5 & 516 & 401 & 363 \\
\hline 6 & 374 & 460 & 433 \\
\hline 7 & 341 & 496 & 481 \\
\hline 8 & 558 & 515 & 511 \\
\hline 9 & 390 & 535 & 541 \\
\hline 11 & 630 & 539 & 548 \\
\hline 12 & 476 & 541 & 552 \\
\hline 13 & 600 & 544 & 555 \\
\hline 15 & 620 & 544 & 556 \\
\hline 16 & 539 & 544 & 557 \\
\hline 17 & 520 & 545 & 557 \\
\hline 18 & 636 & 545 & 557 \\
\hline 19 & 544 & 545 & 557 \\
\hline 20 & 596 & 545 & 557 \\
\hline 21 & & &
\end{tabular}


Figura 2 - Evolução da curva de crescimento para peso de creme comestível de palmito estimada pelos modelos de biomassa e de Gompertz

Figure 2 - Growth curve of the edible heart palm weight generated by the biomass and the Gompertz model

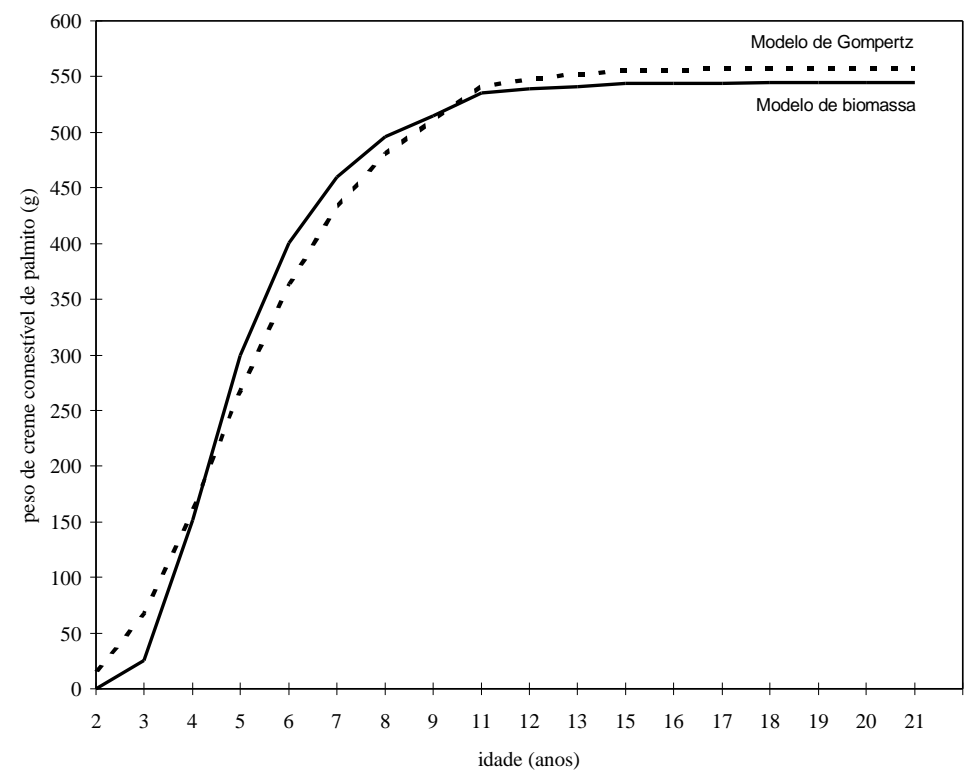

Ao considerar que a determinação da idade em palmiteiros nem sempre é possível, adicionalmente, ajustou-se o Modelo Matemático $\mathrm{Y}=\mathrm{a}+\mathrm{b}$. $\mathrm{X}$ (Equação da Reta), para a predição do diâmetro do creme, em função do DAP, que é a variável que pode ser mensurada com maior facilidade na árvore. Além da facilidade em mensurar o DAP, o valor para o coeficiente de correlação linear simples $(r)$ entre esta variável e o diâmetro do creme, correspondeu a 0,75 e a tendência da dispersão entre as mesmas foi linear, razão pela qual optou-se pela Equação da Reta. Enfatiza-se ainda, que o objetivo principal deste procedimento consiste em obter predições para o diâmetro do creme em função do DAP, e não em preconizar o uso do modelo. Obteve-se os seguintes coeficientes para a Equação da Reta: $a=0,0440252$ e b $=0,3416181$. Os resultados para os indicadores de ajuste foram: $F=745,2575$, $\mathrm{R}^{2}=0,8374$ e Syx $\%=18,46$ e a distribuição dos resíduos para estimativas do diâmetro do creme foi homogênea (ver Anexo).

Em seguida, construiu-se um gráfico (Figura 3), onde compararam-se os valores mensurados com os estimados para o Diâmetro do Creme, e que possibilitou as seguintes constatações:

- A Equação da Reta foi apropriada aos dados, e as predições em função do DAP com dimensões inferiores a $4 \mathrm{~cm}$ foram superestimadas e os DAP superiores ao intervalo considerado, poderão gerar predições absurdas. 
Portanto, as estimativas correspondentes às extremidades do modelo devem ser analisadas com cautela.

- Para o conjunto dos dados, o diâmetro mínimo de $2,5 \mathrm{~cm}$ de creme, corresponde a árvores com DAP superior a $7 \mathrm{~cm}$.

Figura 3 - Distribuição dos diâmetros individuais do creme de palmito: valores observados e estimados pela equação da reta

Figure 3 - Observed and predicted values of diameter of heart palm by the straight line model

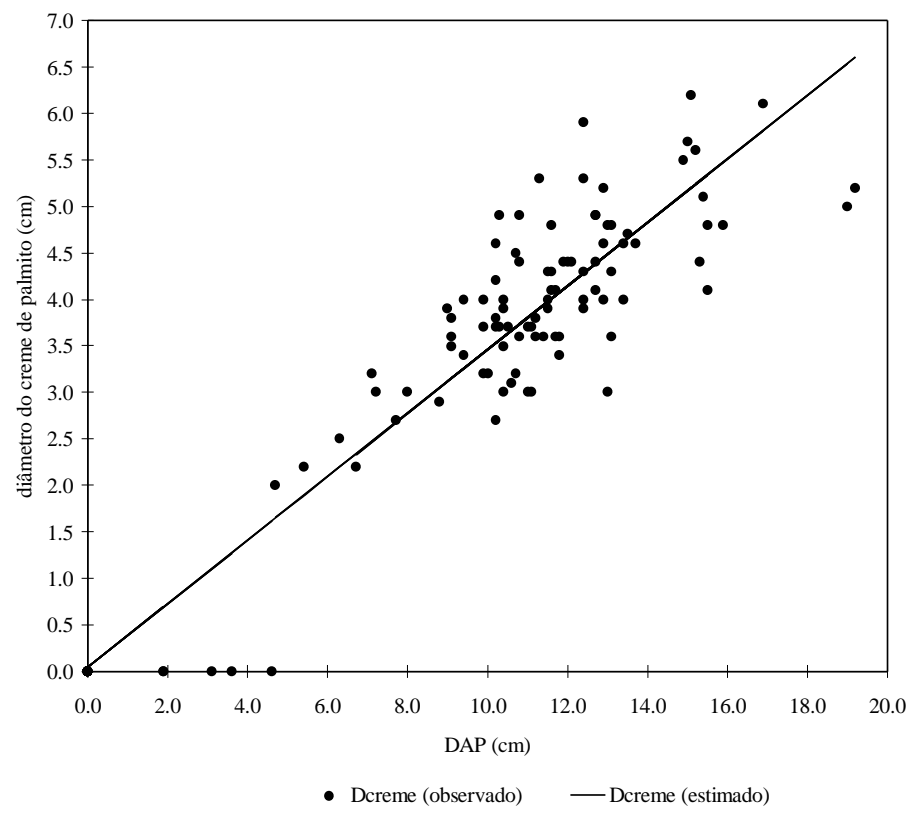

Os valores observados para o Diâmetro do Creme (Dcreme), e as estimativas obtidas pelo Modelo de Gompertz e pela equação da reta, apresentados na Tabela 6, foram comparados. Verificou-se que as predições oriundas dos modelos ajustados pouco diferiram entre si, e também tiveram pequena variação em relação à média observada, (máximo $9 \mathrm{~mm}$ ). Ainda, para a série de dados, o diâmetro de creme $\operatorname{com} 2,5 \mathrm{~cm}$, somente ocorrerá em idades acima de 4 anos, e correspondendo a árvores com o DAP superior a $7 \mathrm{~cm}$.

As estimativas para o diâmetro do creme de palmito, obtidas pelo Modelo de Gompertz, para os dados em análise, tenderam a ser subestimadas em relação às observações, principalmente nas idades mais avançadas (a partir de 16 anos). Este fato pode ser devido à impossibilidade do controle sobre o material disponível e utilizado na pesquisa, tais como: dificuldade em precisar a idade biológica verdadeira das árvores, controle do sítio e do material genético, 
diferenças entre as quantidades de indivíduos disponíveis em cada idade (que estão afetando a média) e eventuais problemas do modelo.

Tabela 6 - Diâmetro do creme de palmito: valores preditos pela equação da reta, modelo de Gompertz e médias observadas por idade

Table 6 - Diameter of heart palm observed and predicted by the straight line model, Gompertz model

\begin{tabular}{c|c|c|c|c}
\hline $\begin{array}{c}\text { Idade } \\
\text { (anos) }\end{array}$ & $\begin{array}{c}\text { DAP } \\
(\mathbf{c m}) /\end{array}$ & \multicolumn{3}{|c}{$\begin{array}{c}\text { Diâmetro do Creme de Palmito } \\
\text { (cm) }\end{array}$} \\
\cline { 3 - 5 }$\left(\begin{array}{c}\text { (years) } \\
(\boldsymbol{c m})\end{array}\right.$ & $\begin{array}{c}\text { Equação da Reta / } \\
\text { Straight line model }\end{array}$ & $\begin{array}{c}\text { Médias observadas / } \\
\text { Observed means }\end{array}$ & $\begin{array}{c}\text { Modelo de Gompertz / } \\
\text { Gompertz model }\end{array}$ \\
\hline 2 & 0,0 & 0,0 & 0,0 & 0,0 \\
\hline 3 & 1,5 & 0,6 & 0,0 & 0,2 \\
\hline 4 & 7,2 & 2,5 & 2,7 & 2,4 \\
\hline 5 & 10,6 & 3,7 & 3,5 & 3,8 \\
\hline 6 & 11,7 & 4,0 & 4,1 & 4,1 \\
\hline 7 & 12,0 & 4,1 & 3,7 & 4,2 \\
\hline 8 & 12,1 & 4,2 & 3,5 & 4,2 \\
\hline 9 & 12,1 & 4,2 & 4,2 & 4,2 \\
\hline 11 & 12,1 & 4,2 & 3,9 & 4,2 \\
\hline 12 & 12,1 & 4,2 & 4,5 & 4,2 \\
\hline 13 & 12,1 & 4,2 & 4,0 & 4,2 \\
\hline 15 & 12,1 & 4,2 & 4,8 & 4,2 \\
\hline 16 & 12,1 & 4,2 & 4,5 & 4,2 \\
\hline 17 & 12,1 & 4,2 & 4,4 & 4,2 \\
\hline 18 & 12,1 & 4,2 & 5,1 & 4,2 \\
\hline 19 & 12,1 & 4,2 & 4,8 & 4,2 \\
\hline 20 & 12,1 & 4,2 & 4,5 & 4,2 \\
\hline 21 & 12,1 & 4,2 & 4,9 & 4,2 \\
\hline
\end{tabular}

\section{CONCLUSÕES E RECOMENDAÇÕES}

O exame dos resultados obtidos neste estudo, permitiu documentar algumas importantes conclusões e recomendações, quais sejam:

- O crescimento e a produção em árvores individuais de E. edulis Mart. pode ser representado através de uma equação matemática como função da idade, e para a série de dados estudada, o modelo mais recomendado foi o de Gompertz.

- A idade mantém relação com o crescimento e a produção, quer seja em diâmetro e altura, ou mesmo em biomassa para Euterpe edulis Mart.. 
Entretanto, a realização de estudos mais aprofundados é recomendada, estabelecendo-se registros de controle mais estritos sobre esta variável, e também em condições de sítio homogêneas.

- A utilização do Modelo de Gompertz em plantios comerciais de palmito, poderá representar satisfatoriamente o crescimento desta espécie, propiciando estimativas realistas acerca da produção desejada de creme de palmito o mesmo podendo ser dito em relação ao Modelo de Chapman-Richards. Contudo, recomenda-se o desenvolvimento de pesquisas para aprofundar estudos sobre as relações biológicas existentes entre as variáveis dimensionais pertencentes a árvores da espécie $E$ edulis Mart., visando desenvolver modelos matemáticos específicos capazes de propiciar melhor representação do crescimento e da produção da espécie.

- Com a série de dados estudada, conclui-se que a culminação do DAP e do diâmetro do creme, ocorre na mesma idade (8 anos), e após a estabilização do crescimento destas variáveis, o peso do creme ainda continuou aumentando. $\mathrm{O}$ diâmetro mínimo de $2,5 \mathrm{~cm}$ de creme comestível será atingido em idades acima de 4 anos, em árvores com DAP superior a 7,5 cm, cujo peso estimado em creme comestível corresponde a $152 \mathrm{~g}$.

- Diante disto, sugere-se que o corte de palmito se inicie após a palmeira atingir pelo menos $10 \mathrm{~cm}$ de DAP, época em que o porte comercial do palmito começará a estar mais propício à industrialização (acima de $3,5 \mathrm{~cm}$ ). Contudo, é importante considerar que em se pensando em regeneração natural, é necessário considerar um lapso maior de tempo, em virtude da frutificação nessa fase ainda não ser regular.

\section{BIBLIOGRAFIA CITADA}

AHRENS, S. Modelos de crescimento e de produção. Trabalho de tópico especial do Curso de Pós-Graduação em Engenharia Florestal. Curitiba: Universidade Federal do Paraná. 1990. 46p., não publicado.

AVERY, E.T. \& BURKHART, E.H. Forest Measurements. New York: MacGrawHill, 1983. 331p.

CARVALHO, P.E.R. Espécies florestais brasileiras: recomendações silviculturais, potencialidades e usos da madeira Colombo : EMBRAPA - CNPFlorestas, 1994. p 246-250.

FANTINI, A.C.; NODARI, R.O.; REIS, M.S.; MANTOVANI, A.; ODORIZZI, J.; RIBEIRO, R.J. Estimativa da produção de palmito em plantas de palmiteiro (Euterpe edulis Martius) a partir de características fenotípicas. Viçosa: Revista Árvore, v.1, n.21, Viçosa-MG, 1997. p. 49-57. 
FERREIRA, V.L.P. \& PASCOALINO, J.E. Pesquisa sobre palmito no Instituto de Tecnologia de Alimentos - ITAL. In: ENCONTRO NACIONAL DE PESQUISADORES EM PALMITO, 1., Colombo, 1987. Anais. Curitiba: EMBRAPA - CNPF, 1987. P.45-63.

HERING, G.K. A mata nativa brasileira: equilíbrio ecológico e produção de celulose. São Paulo: Universidade de São Paulo, 1984. 140p.

HERING, G.K. Manejo florestal. Insula, n.14, p. 162-180, 1984.

HERING, G.K. Diversidade e manejo da Floresta Atlântica Paralelo 27, Florianópolis, 1994. 86p.

KIRCHNER, F.F.; LOZOYA, J.C.R.; OHLSON, J.C. Aspectos quantitativos na estimativa do peso e distribuição por classe de qualidade de palmito (Euterpe edulis). In: ENCONTRO NACIONAL DE PESQUISADORES EM PALMITO, 1., Colombo, 1987. Anais. Curitiba: EMBRAPA - CNPF, 1987. p.119-124.

NODARI, R.O.; GUERRA, M.P.; REIS, A.; REIS, M.S. Incremento anual do palmiteiro (Euterpe edulis). In: ENCONTRO NACIONAL DE PESQUISADORES EM PALMITO, 1., Colombo, 1987. Anais. Curitiba: EMBRAPA - CNPF, 1987. p. 175-176.

NODARI, R.O.; REIS, M.S.; GUERRA, M.P. Relação entre parâmetros não destrutivos e rendimento de palmito - estudo preliminar. In: ENCONTRO NACIONAL DE PESQUISADORES EM PALMITO, 1., Colombo, 1987. Anais. Curitiba: EMBRAPA - CNPF, 1987. p.181-183.

PARANÁ. Secretaria de Estado do Desenvolvimento Urbano e do Meio Ambiente. Coletânea de Legislação Ambiental Federal e Estadual, Curitiba, 1990. p.43.

PEREIRA, R.A, \& ARRUDA, V.H. Ajuste Prático de Curvas na Pesquisa Biológica Campinas: Fundação Cargill, 1987. 50p.

REIS. A; NODARI, R.O.; REIS, M.S.; GUERRA, M.P. Rendimento comercial e relações entre características associadas ao volume de Euterpe edulis - avaliação preliminar. In: ENCONTRO NACIONAL DE PESQUISADORES EM PALMITO, 1., Colombo, 1987. Anais. Curitiba: EMBRAPA - CNPF, 1987. p. 149-158.

RIBEIRO, J.H. SOS Palmito. Revista Globo Rural, v.2, n.14, nov. 1986. p.18 - 30.

ROSSETI, C.F.; BERGER, R.; DUARTE, J. Considerações gerais sobre a produção e comercialização do palmito. In: ENCONTRO NACIONAL DE PESQUISADORES 
EM PALMITO, 1., Colombo, 1987. Anais. Curitiba: EMBRAPA - CNPF, 1987. p. 125-136.

SANQUETTA, C.R. Fundamentos Biométricos dos Modelos de Simulação Florestal Curitiba : FUNDAÇÃO DE PESQUISAS FLORESTAIS DO PARANÁ, Série Didática $n^{0} 08$, Curitiba: 1996. 49p.

SPAIN, J.D. Basic Microcomputer Models in Biology. Massachusetts: AddisonWesley Publishing Company, 1982. 354p.

\section{ANEXO}

Distribuição de resíduos dos modelos de crescimento ajustados Graphical distribution of residuals of the growth models

a) Gompertz (altura da estipe / stem height)

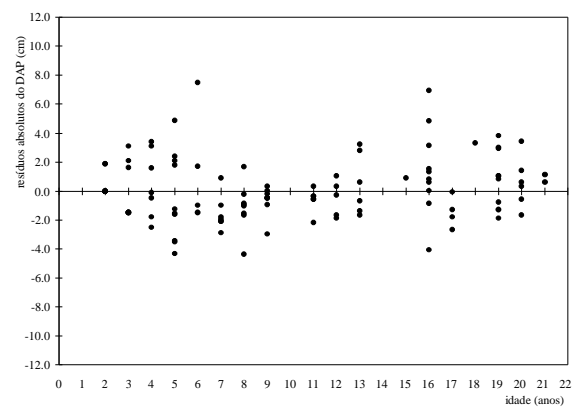

b) Gompertz (DAP / DBH)

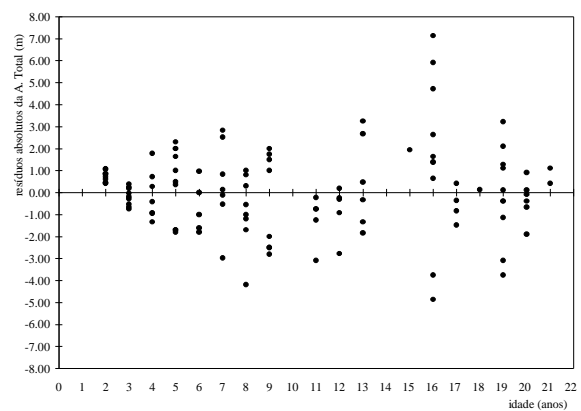


c) Gompertz (altura total / total height)

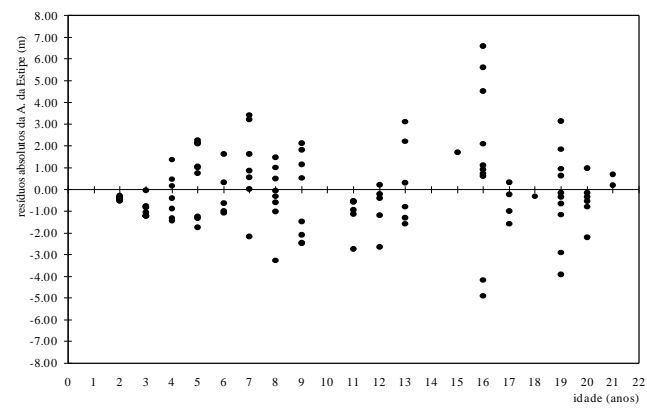

d) Biomassa /Biomass (peso de creme/ edible weight)

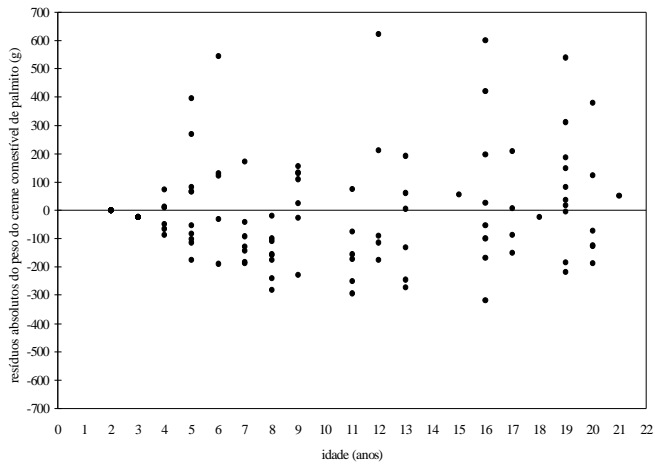

e) Gompertz (diâmetro de creme/ diameter of the edible portion)

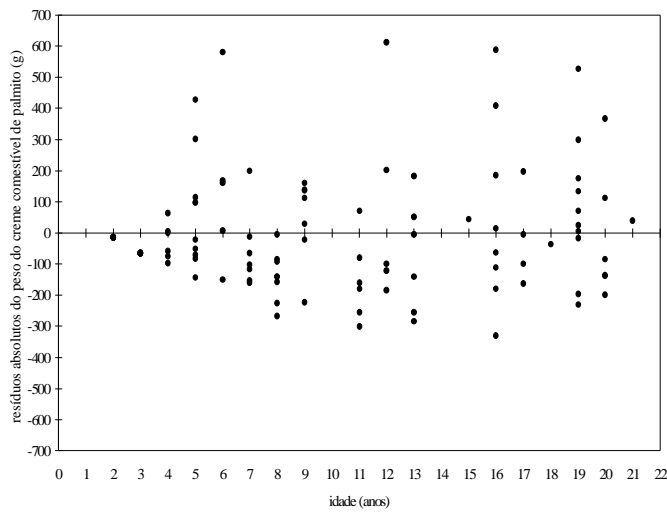


f) Gompertz (peso de creme/ edible weight)

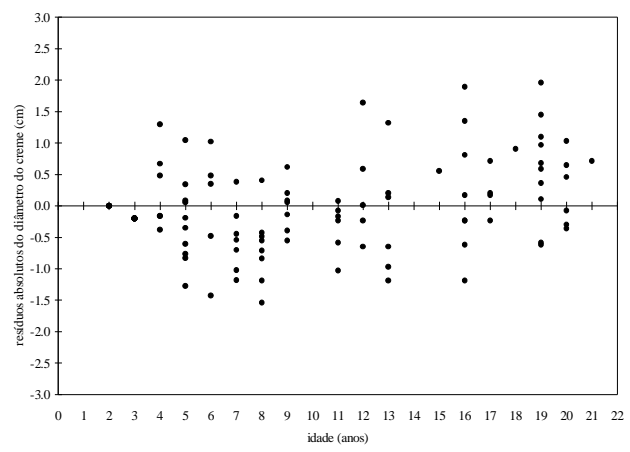

g) Reta / Straight line (diâmetro de creme/ diameter of the edible portion)

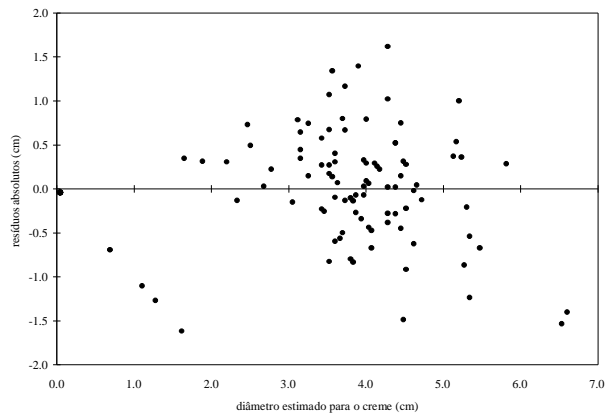

